\title{
Increase in the OCT angiographic peripapillary vessel density by ROCK inhibitor ripasudil instillation: a comparison with brimonidine
}

\author{
Etsuo Chihara ${ }^{1}$ (D) Galina Dimitrova ${ }^{2} \cdot$ Tomoyuki Chihara $^{1,3}$ \\ Received: 28 August 2017 / Revised: 29 December 2017 / Accepted: 27 February 2018 / Published online: 8 March 2018 \\ (C) The Author(s) 2018. This article is an open access publication
}

\begin{abstract}
Purpose To assess the responses of the superficial peripapillary retinal vessel density (VD) and prelaminar flow index (PLFI) to topical Rho-assisted coiled-coil forming protein kinase (ROCK) inhibitor ripasudil and alpha-2 agonist brimonidine using optical coherence tomography angiography.

Methods This is a prospective, non-randomized, comparative cohort study. We studied the response of optical coherence tomography angiography (OCTA) parameters to drugs in 24 eyes treated with ripasudil and 23 eyes treated with brimonidine at the Sensho-kai Eye Institute. After division by the signal strength (SS), we compared the responses of peripapillary VD/SS and $\mathrm{PLFI} /$ unit area (UA)/SS to topical eye drops in eyes with primary open-angle glaucoma (POAG) and ocular hypertension (OH). Results In the superficial peripapillary retina, VD/SS increased significantly in the ripasudil-treated eyes $(12.5 \pm 21.7 \%, P=$ $0.018)$, but not in the brimonidine-treated eyes $(-2.0 \pm 13.8 \%, P=0.484)$. In the deeper area of the optic disc, the changes in the $\mathrm{PLFI} / \mathrm{UA} / \mathrm{SS}$ in the brimonidine-treated eyes $(+0.9 \pm 8.9 \%, P=1.00)$ and ripasudil-treated eyes $(-1.3 \pm 8.5 \%, P=0.241)$ were not significant. Multivariate discriminant analysis showed that the change in the peripapillary VD/SS was the most important parameter $(P=0.0186)$ for differentiating ripasudil- and brimonidine-treated eyes.

Conclusions The topical ROCK inhibitor ripasudil enhanced the peripapillary VD in POAG and OH, whereas the alpha-2 agonist brimonidine did not. The PLFI did not respond to either drug.
\end{abstract}

Keywords OCT angiography $\cdot$ Glaucoma $\cdot$ Radial peripapillary capillary $\cdot$ ROCK inhibitor $\cdot$ Ripasudil $\cdot$ Brimonidine

\section{Introduction}

Vascular insufficiency is one of the main features of glaucomatous optic neuropathy, and fluctuation of the optic disc blood flow is considered a causative factor of nerve loss [1]. Abnormal microcirculation can occur in both the optic

Electronic supplementary material The online version of this article (https://doi.org/10.1007/s00417-018-3945-5) contains supplementary material, which is available to authorized users.

Etsuo Chihara

chihara-oph@umin.net

1 Sensho-kai Eye Institute, Minamiyama 50-1, Iseda, Uji, Kyoto 611-0043, Japan

2 Department of Ophthalmology, City General Hospital 8th September, Skopje, Macedonia

3 Department of Ophthalmology, Kansai Medical University, Hirakata, Osaka, Japan nerve head $(\mathrm{ONH})$ and the superficial peripapillary retinal vasculature $[2,3]$. The results of recent optical coherence tomography angiography (OCTA) studies have confirmed reduced blood flow in glaucomatous ONH [4], a finding reported previously using the laser speckle technique and microsphere method [5]. However, susceptibility to glaucomatous damage may differ based on the location of vessels. The results of OCTA studies have suggested that superficial vessels such as the radial peripapillary capillaries are susceptible to glaucomatous damage [6], while the microcirculation in the prelaminar area might be resistant to glaucomatous insults in the earliest stage of glaucoma [7].

Among the many parameters that affect blood flow in the $\mathrm{ONH}$, topical antiglaucoma medications may improve microcirculation in the optic nerve head.

The Rho-assisted coiled-coil forming protein kinase (ROCK) inhibitor ripasudil (Glanatec ${ }^{\circledR}$, Kowa Co., Ltd., Aichi, Japan) and its analogues have the potential to reduce intraocular pressure (IOP) in clinical practice $[8,9]$ and vasodilate vessels $[10,11]$ and may improve microcirculation 
in the optic disc $[12,13]$. Other than the hypotensive effects, antiglaucoma drugs may suppress oxidative stress [14] and inhibit apoptosis [15]. However, concentration and effects of drugs in and around the ONH after topical instillation have been unknown. If vasodilation of the $\mathrm{ONH}$ vessel, which was not proven in the case of brimonidine [16], was confirmed after topical instillation of ripasudil, that would provide pharmacologic evidence that a sufficiently high drug concentration reached the $\mathrm{ONH}$.

In the current prospective study, we compared the effects of ripasudil and brimonidine on the peripapillary vessel density (VD) and prelaminar blood flow index (PLFI) in patients with glaucoma.

\section{Patients and methods}

This was a prospective, non-randomized, comparative study conducted from September 2015 to March 2017 that included 55 eyes of 55 consecutive patients aged 20 years and older. The patients had poorly controlled intraocular pressure (IOP) or visual field progression, required antiglaucoma medication (ripasudil or brimonidine), and were treated at the outpatient service of the Sensho-kai Eye Institute.

The inclusion criteria included the presence of primary open-angle glaucoma (POAG), preperimetric glaucoma, or ocular hypertension $(\mathrm{OH})$. These patients had not undergone previous retinal surgery or laser photocoagulation. Patients who underwent uneventful cataract surgery or glaucoma surgery more than 12 months prior to enrollment into this study were allowed. Previous use of a topical prostaglandin and carbonic anhydrase was allowed; however, patients who used beta blockers were excluded.

The exclusion criteria included the presence of cloudy media, poor-quality OCTA images characterized by doubling of vessel images and artifact lines in the target area, signal strength (SS) below 40, high pretreatment IOP exceeding $30 \mathrm{mmHg}$, visual acuity (VA) less than 20/40, and mean deviation (MD) below $-20 \mathrm{~dB}$. If vitreous floaters hampered analysis of peripapillary or $\mathrm{ONH}$ images, the eyes were excluded from the study. Other exclusion criteria included glaucoma surgery during the study period, a highly deformed optic disc, extensive myopic peripapillary chorioretinal atrophy, iridocyclitis, congenital anomaly or neuro-ophthalmologic diseases, epiretinal membrane, amblyopia, and/or retinal vascular diseases. Patients were also excluded if they had a renal disease, systemic hypertension exceeding 160/90 mmHg, diabetes mellitus, or heart disease, who required a vasodilatory drug such as nitroglycerin, and those who drank alcohol or coffee within $3 \mathrm{~h}$ before the examination.

POAG was diagnosed based on a gonioscopic open angle, signs of glaucomatous optic neuropathy using ophthalmoscopy and OCT, and the absence of neuro-ophthalmologic diseases that cause optic nerve atrophy. Glaucomatous visual field defects were studied using the Humphrey Field Analyzer (HFA) (750i, Carl Zeiss Meditec, Tokyo, Japan). Preperimetric glaucoma was defined by positive signs of glaucomatous optic neuropathy on the OCT or nerve fiber layer studies without any visual field defects detected by the HFA (normal glaucoma hemifield test and pattern standard deviation less than 95\%). $\mathrm{OH}$ was defined by the presence of an open angle and a history of at least two repeated IOP values exceeding $21 \mathrm{mmHg}$ but no glaucomatous visual field defects or glaucomatous optic neuropathy.

We recorded the patients' age, spherical equivalent refractive error, best-corrected VA, gonioscopy, slit-lamp microscopy, Goldmann applanation tonometric IOP, and the MD and pattern standard deviation of the Humphrey 24-2 visual field test. The presence of systemic hypertension $(140 / 90 \mathrm{mmHg}$ by the Japanese Society of Hypertension Guideline 2014), diabetes mellitus (fasting blood sugar $>126 \mathrm{mg} / \mathrm{dl}$, hemoglobin A1C > 6.5\%, Japan Diabetes Society Guideline 2012), and cardiac diseases (angina pectoris, myocardial infarction, arrhythmia) was checked by careful history taking. Twentyfour normal subjects had normal visual fields detected by the HFA.

Allocation of drugs Drugs were prescribed in the following order: ripasudil, brimonidine, and then ripasudil.

Masking Four experienced technicians who did not know the clinical background of the patients obtained images and uploaded them to the filing system of the Sensho-kai Eye Institute. One investigator (T.C.) who was independent of the examiners recorded numerical data, and the other independent investigator (E.C.) analyzed the numerical data.

When both eyes of a patient were examined, the eyes with better-quality images were enrolled in the study. If both eyes were eligible, the right eye was selected.

The intravisit reproducibility of data was assessed by repeating examinations five times in each of five normal subjects who had no history of intraocular surgery, diabetes mellitus, systemic hypertension, or glaucomatous optic neuropathy and had an IOP below $21 \mathrm{mmHg}$.

OCT study Patients with glaucoma and $\mathrm{OH}$ were examined twice before eye drops were prescribed and two times between 1 and 2 months after the initiation of eye drop treatment; the mean data were analyzed. If the OCTA images obtained during one of the two examinations were poor, a third examination was performed. The compliance of patients with eye drop instillation was confirmed by patient inquiry. The methodology of obtaining OCTA images was previously reported [7]. Briefly, the enrolled eyes were studied using spectral-domain OCT equipped with an angiographic function (2014.2.0.90 software version) (AngioVue, RTVue XR OCT, Optovue, 
Fremont, CA, USA), and 4.5 × 4.5-mm images were obtained. The parameters studied were the global loss volume (GLV) of the ganglion cell complex, circumpapillary retinal nerve fiber layer thickness (cpNFLT), signal strength (SS), vessel density (VD) by measuring 0 to $80 \mu \mathrm{m}$ from the internal limiting membrane within $700 \mu \mathrm{m}$ of the disc margin, and prelaminar vascular flow index (PLFI). To study the PLFI, Elschnig's scleral ring was marked, the vessels in the cylindrical column 50 to $250 \mu \mathrm{m}$ from the surface, which included the prelaminar area, were highlighted, and the mean decorrelation was measured as the flow index [17]. To avoid the effect of disc size, the PLFI was divided by the area measured (in square millimeters), i.e., the PLFI/unit area (UA) was used for the analysis.

The wavelength of the OCTA device was $840 \mathrm{~nm}$. The definition of the GLV was reported elsewhere [18]. Elimination of projection artifacts was based on the slab subtraction algorithm [19].

The VD was defined as the percentage of signal-positive pixels/area of interest. The algorithm to detect the disc margin was reported previously [20]. Considering patient-specific media opacity, tissue vascularity, refractive error, and waning of the SS, the VD/SS and PLFI/UA/SS were also evaluated.

The mean perfusion pressure (PP) was defined by the formula of Yaoeda et al., where $\mathrm{PP}=2 / 3$ (diastolic blood pressure $[\mathrm{BP}]+1 / 3$ (systolic BP - diastolic BP) - IOP) [21].

The institutional review board of the Sensho-kai Eye Institute approved the study design. All subjects provided informed consent after they received an explanation of the study nature and possible consequences. The study adhered to the tenets of the Declaration of Helsinki.

Statistical analysis The Bell Curve Excel Tokei 2016 (SSRI, Tokyo, Japan) was used to assess data by Wilcoxon signedrank test, Mann-Whitney $U$ test, chi-squared test, Tukey's multiple comparison, Pearson's correlation coefficient, and multivariate discriminant analysis.

The sample size was calculated based on the effective size and power of the $\Delta \mathrm{VD} / \mathrm{SS}$. The power to calculate the difference between the brimonidine and the ripasudil cohorts to the level of $5 \%$ significance was 0.95 for two-sided data analysis. The appropriate sample size to obtain a significance level of 0.05 in two-sided paired data was 23 .

\section{Results}

One eye of 55 patients ( 27 assigned to brimonidine and 28 to ripasudil) and one eye of 24 normal subjects were enrolled in this study; eight patients were excluded after enrollment (one patient because of poor compliance, three because of diabetes mellitus diagnosed later, three with poor-quality images, and one with high systemic BP during the follow-up periods).

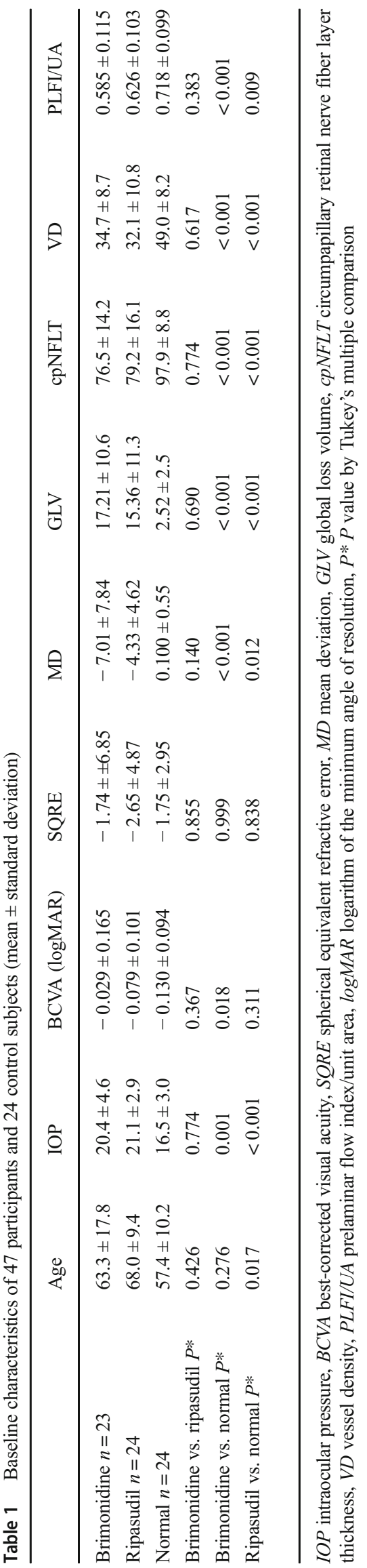


Ultimately, 24 eyes of 24 patients assigned to ripasudil and 23 eyes of 23 patients assigned to brimonidine were included. Thirty-six eyes had POAG (including two with preperimetric glaucoma), and 11 eyes had $\mathrm{OH}$.

Nine eyes had new prescriptions, and 15 eyes had added-on prescriptions of ripasudil, respectively; the numbers for brimonidine were 14 and nine eyes, respectively, $(P=0.11$, by the chi-squared test).

In five normal subjects, the intravisit reproducibility of the data for the SS, VD, PLFI, VD/SS, PLFI/UA, and PLFI/UA/ $\mathrm{SS}$ was assessed by repeating examinations five times. The mean and standard deviation of the coefficient of variation of each parameter were $4.3 \pm 1.6,3.4 \pm 3.1,6.3 \pm 2.7,2.7 \pm 1.6$, $6.6 \pm 3.9$, and $7.6 \pm 3.7 \%$, respectively.

Table 1 shows the demographic data of the participants. There were no significant differences in age, IOP, bestcorrected visual acuity, spherical equivalent refractive error, MD, GLV, cpNFLT, VD, or PLFI/UA $(P=0.426,0.774$, $0.367,0.855,0.140,0.690,0.774,0.617$, and 0.383 , respectively) between the brimonidine and the ripasudil cohorts by Tukey's multiple comparison.

Table 2 shows the changes in the IOP and perfusion pressure by eye drop instillation. The IOP decreased significantly by $12.1 \pm 14.8 \%(P<0.001)$ in the ripasudil-treated eyes and $21.8 \pm 12.6 \%(P<0.001$ Wilcoxon signed-rank test $)$ in the brimonidine-treated eyes. The IOP after brimonidine treatment was significantly ( $P=0.0103$, Mann-Whitney $U$ test) lower than in the ripasudil-treated eyes (Table 2). The baseline PP values in the ripasudil-and brimonidine-treated cohorts were $51.7 \pm 10.0$ and $48.6 \pm 13.3 \mathrm{mmHg}$, respectively. The post-treatment PP values of $52.8 \pm 11.1 \mathrm{mmHg}$ in the ripasudil-treated cohort did not differ significantly from that of the baseline $(P=0.100)$; however, the post-treatment PP of $51.3 \pm 7.9 \mathrm{mmHg}$ in the brimonidine-treated cohort was significantly higher than that of baseline $(P=0.0023$ Wilcoxon signed-rank test). Changes in GLV or cpNFLT by brimonidine or ripasudil treatment were not significant $(P=0.211-0.879$; appendix Table 4).

The responses of the OCTA parameters to brimonidine and ripasudil are shown in Table 3 . Increases in the peripapillary VD $(22.9 \pm 40.0 \%, P=0.025)$ and $\mathrm{VD} / \mathrm{SS}$
$(12.5 \pm 21.7 \%, P=0.018)$ by ripasudil were significant by Wilcoxon signed-rank test; however, changes in these parameters in the brimonidine-treated cohort $(1.8 \pm 23.2 \%$, $P=0.903$ and $-2.0 \pm 13.8 \% P=0.484)$ were not significant. Changes in SS, PLFI/UA, and PLFI/UA/SS in brimonidine-treated eyes $(2.8 \pm 11.7 \%, P=0.162 ; 4.0 \pm$ $15.8 \%, P=0.248 ; 0.9 \pm 8.9 \%, P=1.0$, respectively) and in ripasudil-treated eyes $(8.1 \pm 16.0 \%, P=0.098 ; 5.6 \pm$ $14.0 \%, P=0.241$; and $-1.3 \pm 8.5 \%, P=0.241$, respectively) were not significant (Table 3 ). When the percentage change of the OCTA parameters was compared between brimonidine-and ripasudil-treated cohorts, only the differences in VD $(P=0.035)$ and $\mathrm{VD} / \mathrm{SS}(P=0.033)$ were significant by Mann-Whitney $U$ test (Fig. 1).

Figure 2 shows a representative case, who shows increased VD/SS despite stationary PLFI/UA after ripasudil treatment (Fig. 2a-d) and stationary VD/SS and PLFI/UA after brimonidine treatment (Fig. 2e, g). Horizontal section images of Fig. 2c, $d$ are shown in the appendix (Figs. $2 \mathrm{~g}$ and $2 \mathrm{~h}$ ).

The Pearson's correlation coefficients between $\Delta \mathrm{VD} / \mathrm{SS}$ and $\triangle \mathrm{PP}$ and $\triangle \mathrm{IOP}$ were $r=-0.304(P=0.251)$ and $r=-$ $0.205(P=0.446)$, respectively, for the brimonidine cohort, $r=-0.340(P=0.134)$ and $r=0.205(P=0.386)$, respectively, for the ripasudil cohort, and were not significant in this study.

The associations between the post- and pretreatment differences in IOP, VD/SS, PLFI/UA/SS, GLV, and cpNFLT and the drugs were analyzed by multivariate analysis (discriminant analysis with a method of increasing and decreasing variables). When the $\Delta \mathrm{IOP}, \Delta \mathrm{VD} / \mathrm{SS}, \Delta \mathrm{PLFI} / \mathrm{UA} / \mathrm{SS}, \Delta \mathrm{GLV}$, and $\triangle$ cpNFLT were independent parameters and the use of ripasudil and brimonidine was set as a dependent parameter, the most significant parameter that differentiated ripasudiland brimonidine-treated eyes was $\triangle \mathrm{VD} / \mathrm{SS}(P=0.0186)$ followed by $\triangle \mathrm{IOP}(P=0.099), \Delta \mathrm{cpNFLT}(P=0.249)$, $\triangle \mathrm{GLV}(P=0.384)$, and $\triangle \mathrm{PLFI} / \mathrm{UA} / \mathrm{SS}(P=0.454)$. Because the significantly effective parameters for calculating the coefficient of discrimination were selected at the $P=0.2$ level (Bell Curve Tokei Manual), the obtained discriminant equation for calculating discriminative coefficient $\mathrm{A}$ was $A=9.39 \Delta \mathrm{VD} / \mathrm{SS}+0.183 \Delta \mathrm{IOP}-0.528$, and its

Table 2 Changes in studied parameters by topical eye drops

\begin{tabular}{|c|c|c|c|c|c|c|c|c|}
\hline & Baseline IOP & Post-treatment IOP & $P^{*}$ & $\%$ change & Baseline PP & Post-treatment PP & $P^{*}$ & $\%$ change \\
\hline Brimonidine $n=23$ & $20.4 \pm 4.6$ & $15.7 \pm 3.3$ & $<0.001 * *$ & $-21.8 \pm 12.6$ & $48.6 \pm 13.3$ & $51.3 \pm 7.9$ & $0.0023 * *$ & $15.2+28.8$ \\
\hline Ripasudil $n=24$ & $21.1 \pm 2.9$ & $18.3 \pm 3.0$ & $<0.001 * *$ & $-12.1 \pm 14.8$ & $51.7 \pm 10.0$ & $52.8 \pm 11.1$ & 0.1005 & $3.7 \pm 16.5$ \\
\hline$P$ & 0.983 & $0.0103 *$ & & $0.0086^{* *}$ & 0.315 & 0.615 & & 0.14 \\
\hline
\end{tabular}

$P^{*}=P$ value (Wilcoxon signed-rank test) for difference between baseline and post-treatment. $\mathrm{P}=P$ value (Mann-Whitney $U$ test) for comparison between brimonidine- and ripasudil-treated cohorts

$I O P$ intraocular pressure $(\mathrm{mmHg}), P P$ perfusion pressure $(\mathrm{mmHg})$

*Significant with $P<0.05$; **Significant with $P<0.01$ 
Table 3 Responses of optical coherence tomography angiography parameters to brimonidine and ripasudil treatments

\begin{tabular}{lcccc}
\hline & Baseline & Post-treatment & $\%$ changes & $P$ \\
\hline $\begin{array}{l}\text { Signal strength (SS) } \\
\text { Brimonidine }\end{array}$ & $56.0 \pm 9.2$ & & & \\
Ripasudil & $55.4 \pm 8.7$ & $57.1 \pm 7.9$ & $102.8 \pm 11.7$ & 0.162 \\
Vessel density (VD) & & $59.1 \pm 7.0$ & $108.1 \pm 16.0$ & 0.098 \\
Brimonidine & $34.7 \pm 8.7$ & & & \\
Ripasudil & $32.1 \pm 10.8$ & $34.8 \pm 9.2$ & $101.8 \pm 23.2$ & 0.903 \\
VD/SS & & $36.7 \pm 8.7$ & $122.9 \pm 40.0$ & $0.025^{*}$ \\
Brimonidine & $0.614 \pm 0.083$ & $0.602 \pm 0.112$ & $98.0 \pm 13.8$ & 0.484 \\
Ripasudil & $0.568 \pm 0.125$ & $0.620 \pm 0.093$ & $112.5 \pm 21.7$ & $0.018^{*}$ \\
Prelaminar flow index (PLFI)/unit area (UA) & & & \\
Brimonidine & $0.585 \pm 0.115$ & $0.603 \pm 0.125$ & $104.0 \pm 15.8$ & 0.248 \\
Ripasudil & $0.626 \pm 0.103$ & $0.650 \pm 0.064$ & $105.6 \pm 14.0$ & 0.241 \\
PLFI/UA/SS & & & & \\
Brimonidine & $0.0105 \pm 0.0017$ & $0.0106 \pm 0.0017$ & $100.9 \pm 8.9$ & 1 \\
Ripasudil & $0.0113 \pm 0.0012$ & $0.0111 \pm 0.0010$ & $98.7 \pm 8.5$ & 0.241 \\
\hline
\end{tabular}

$P$ : by Wilcoxon signed-rank test to compare between baseline and post-treatment data $V D / S S$ vessel density/signal strength, PLFI/UA prelaminar flow index/unit area, PLFI/UA/SS prelaminar flow index/unit area/signal strength

*Significant $P<0.05$

discriminative power was $59.1 \%$ for brimonidine and $65.2 \%$ for ripasudil. The centers of the discriminative coefficient for brimonidine and ripasudil were 0.4464 and -0.4270 , respectively (Fig. 3 ).

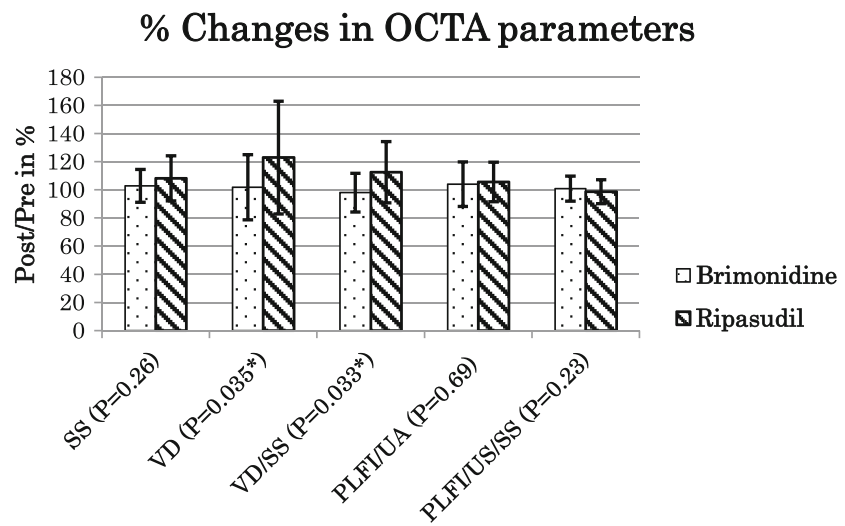

Fig. 1 Changes in optical coherence tomography angiography parameters by brimonidine (dotted columns) and ripasudil (striped column) treatment. The increases in the vessel density (VD) and VD/ signal strength (SS) in the ripasudil-treated cohort were significant by $22.9 \pm 40 \%(P=0.025)$ and $12.5 \pm 21.5 \%(P=0.018$ by Wilcoxon signed-rank test), respectively, while those in the brimonidine-treated eyes of $1.8 \pm 23.2 \%(P=0.903)$ and $-2.0 \pm 13.8 \%(P=0.484$ by Wilcoxon signed-rank test), respectively, were not significant (Table 3). There were significant differences in the percentage increase in the VD and VD/SS between ripasudil- and brimonidine-treated cohorts $(P=$ 0.035 and $P=0.033$, respectively) by the Mann-Whitney $U$ test. On the other hand, the SS, prelaminar flow index/unit area (PLFI/UA) and the $\mathrm{PLFI} / \mathrm{UA} / \mathrm{SS}$ did not change after brimonidine or ripasudil treatment, and there was no difference between ripasudil- and brimonidine-treated cohorts by $P=0.26, P=0.69$, and 0.23 , respectively ( $P$ values, by Mann-Whitney $U$ test).

\section{Discussion}

Many studies have been published concerning improved microcirculation in the $\mathrm{ONH}$ resulting from topical antiglaucoma medications. Other than the direct effects of the drugs, the reduced IOP itself [22] and the systemic BP or PP might affect the optic disc or retinal perfusion $[23,24]$. The combination of high IOP and low systolic BP led to dysregulation of the optic disc flow in rhesus monkeys [25]. In the current study, associations between $\Delta \mathrm{VD} / \mathrm{SS}$ and $\Delta \mathrm{PP}$ and $\Delta \mathrm{IOP}$ were not significant in the ripasudil-treated patients $(P=0.134$ and $P=$ 0.386 , respectively). The reduction in the IOP was more pronounced in the brimonidine-treated cohort $(21.8 \%)$ than in the ripasudil-treated cohort $(12.1 \%)$, and the increase in the PP was significant only in the brimonidine-treated cohort $(P=$ 0.0023 Table 2 ). Despite a theoretically favored hemodynamic situation in the brimonidine-treated eyes, an increase in the peripapillary VD was noted only in the ripasudil cohort. Therefore, the effects of the PP and IOP on optic disc microcirculation may be less than the pharmacological effects of ripasudil.

ROCK inhibitors disrupt actin filaments and inhibit contraction of the smooth muscle [26, 27]. Fasudil, an analogue of ripasudil, has a potent vasodilatory effect and is clinically used to treat arteriosclerosis, vasospasm, and hypertension $[10,11]$. The effect of ripasudil is 5 to 10 times that of fasudil $[14,28]$, and an intravitreal injection of ripasudil increased the retinal blood flow in experimental studies [29]. Thus, if the concentration of ripasudil is sufficiently high, vasodilation might occur in the ONH. Some reports have suggested increased blood 

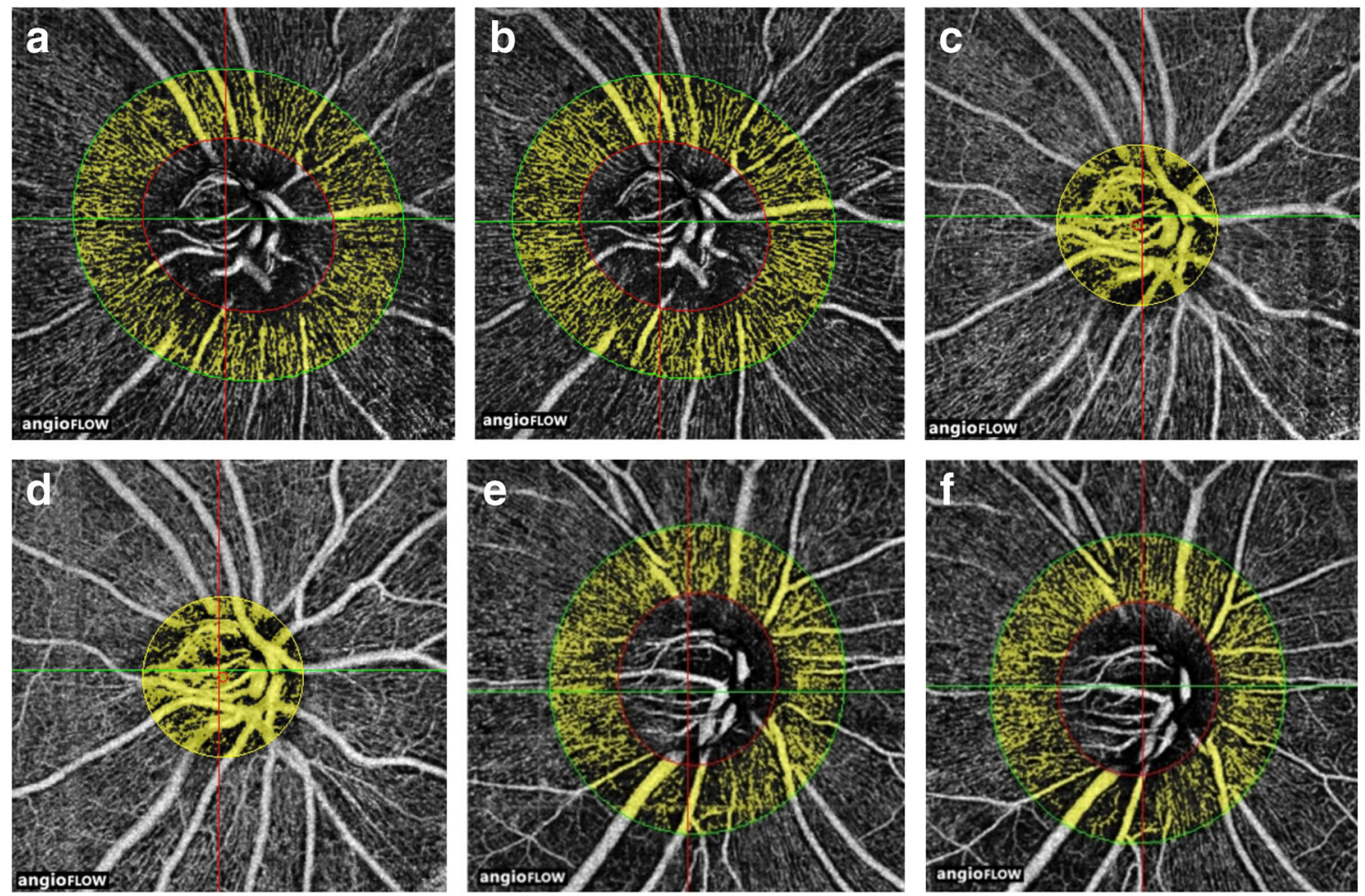

Fig. 2 Increased peripapillary vessel density (VD) after ripasudil treatment (b) in comparison to baseline VD (a). This finding is in contrast to stationary vasculature at the prelaminar area in the same eye (c baseline and $\mathbf{d}$ after treatment) and stationary peripapillary vessel density after brimonidine treatment (e baseline and $\mathbf{f}$ after treatment). a Baseline superficial peripapillary vessels before ripasudil treatment. The vessel density/signal strength $(\mathrm{VD} / \mathrm{SS})$ was $0.741(\mathrm{VD}=43 ; \mathrm{SS}=58)$. The highlighted area is $700 \mu \mathrm{m}$ from the disc margin. b Peripapillary VD after ripasudil treatment. Peripapillary VD and VD/SS increased from 43 and 0.741 to 50 and 0.820 (increase of 16.2 and $10.7 \%$, respectively) after start of ripasudil treatment. This increase was significant (Fig. 1 and Table 3). SS was 61 for this image. c Baseline prelaminar area vessels $(50-250 \mu \mathrm{m}$ from the surface) examined at the same time as a acquisition. The PLFI/UA and PLFI/UA/SS before ripasudil treatment were 0.630 and 0.0109 , respectively $(\mathrm{PLFI}=1.242$,

flow in the ONH resulting from topical instillation of a ROCK inhibitor [12,13].

Interestingly, the effect of ripasudil was found in the OCT angiographic superficial peripapillary area and not in the prelaminar area. If penetration of ripasudil occurs via the periocular transscleral and/or uveoscleral routes [30], the ripasudil concentration might be higher in the peripapillary retina than in the $\mathrm{ONH}$ and cause vasodilation of only the peripapillary vessels [31]. The presence of Kuhnt intermediary tissue as a barrier between the choroid and the $\mathrm{ONH}$ might hamper drug diffusion from the choroid or sclera to the $\mathrm{ONH}$ [32]. Different responses in the peripapillary vessels and prelaminar area vessels to the drugs, IOP, or glaucomatous insults might be another reason [33, 34].

The current results suggest that ripasudil reaches the $\mathrm{ONH}$ in a sufficiently high concentration to vasodilate the peripapillary vessels. However, the association between area examined $=1.97 \mathrm{~mm}^{2}, \mathrm{SS}=58$ ). $\mathbf{d}$ Stationary prelaminar vessels (deeper vessels of $\mathbf{b}$ ) after ripasudil treatment started. In contrast to the superficial peripapillary vessels, the post-treatment PLFI/UA (0.670) and $\mathrm{PLFI} / \mathrm{UA} / \mathrm{SS}(0.0110)\left(\mathrm{PLFI}=1.333\right.$, area examined $\left.=1.99 \mathrm{~mm}^{2}, \mathrm{SS}=61\right)$ did not change much from the baseline of 0.630 and 0.0109 , which was increased +6.3 and $+0.9 \%$, respectively. When percentile changes in PLFI/UA and PLFI/UA/SS were compared between ripasudil- and brimonidine-treated cohorts, differences were not significant $(P=0.69$ and 0.23 , respectively, by Mann-Whitney $U$ test: Fig. 1 and Table 5 in appendix). e Baseline peripapillary superficial vessels before brimonidine treatment started. f Stationary peripapillary vessel density after topical instillation of brimonidine started. The VD/signal strength of 0.690 after brimonidine treatment started did not change markedly from the pretreatment level of 0.700

vasodilation and oxidative stress or neuroprotection is unknown. Clinical evidence that supports neuroprotection of the optic nerve has not yet been reported for ripasudil and is a subject for future studies.

Improvement of the blood flow by brimonidine was not confirmed in this study or in a previous study [16]. Therefore, the neuroprotective effect of brimonidine, if it exists [35], might not be attributed to changes in the microcirculation but to other mechanisms such as modification of Bcl-2 [15].

The flow index in the current study was defined as an average decorrelation at the segmented area [20]. The correlation between the decorrelation and the true blood flow is not parallel but is a sigmoid association [36]; thus, the flow index should be considered one of the indicators of blood flow. Because noise below the level of 0.125 was cut and the blood flow signal from the large vessels was saturated, the changes 


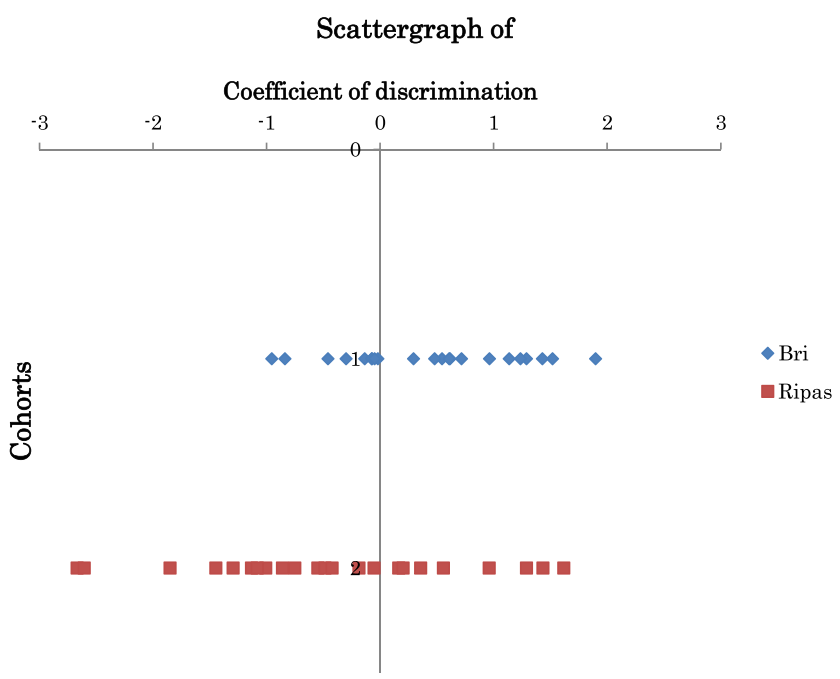

Fig. 3 Scatter graph of the discriminant coefficient $A(A=9.39 \Delta \mathrm{VD} /$ $\mathrm{SS}+0.183 \Delta \mathrm{IOP}-0.528)$. Multivariate analysis showed that the change in $\Delta \mathrm{VD} / \mathrm{SS}(P=0.0186)$ is the most important parameter of the coefficient for discrimination between brimonidine- and ripasudiltreated cohorts. The center of the coefficient for brimonidine-treated eyes was 0.4464 and that of ripasudil was -0.4270 . Bri brimonidine, Ripas ripasudil. Dots are discriminant coefficient $(A)$ of each patient

in the flow index in the current study are expected to reflect the capillary level blood flow changes. In our previous study, the flow index was correlated closely with the VD (Pearson's correlation coefficient of 0.93 to 0.99 [24]) and might be considered as a parameter that is nearly identical to the VD.

Study limitations In this study, segmentation of the prelaminar and laminar tissue is poor, and the PLFI covers a large part of the prelaminar area and a small part of the lamina cribrosa. However, the poor segmentation between the lamina and the prelaminar region may not affect the result much, because in an experimental setting, the blood flow at the laminar tissue did not differ significantly from that in the prelaminar area [37]. For future studies, precise segmentation of the lamina cribrosa is desirable to elaborate the pathophysiology at the prelaminar area.

When the OCT angiographic quantitative data are analyzed, care must be taken regarding the limits in the ability of OCT angiography to assess blood flow and modification factors. Current OCT angiography technology is still immature to assess the blood flow. This may be the reason why the OCT angiographic blood flow parameters are not validated well and need further improvement. The type of software, algorithm to reduce projection artifacts and machine used, severity of glaucoma, refractive error, age, and retinal thickness may affect the VD [7]. Other than the parameters studied in this report, diabetes mellitus, renal function, systemic drugs, and others might affect the results and are subjects for future studies.

In conclusion, topical ripasudil enhanced the VD of the peripapillary superficial retina but not the PLFI. This finding differed from the response in cases treated with topical brimonidine, in which both the peripapillary and the prelaminar area microcirculation remained unchanged.

Acknowledgments Participating investigators: data collected by Ms. Nakatani K, Abe F, Kajimoto E, Morigaki T, Nishimura M, Hashimoto N, Suhara C, Oku Y, Kinoshita C, Matsushita A, and Nishimura R from the Sensho-kai Eye Institute.

\section{Compliance with ethical standards}

Conflict of interest The authors declare that they have no conflict of interest.

Ethical approval All procedures performed in studies involving human participants were in accordance with the ethical standard of the institutional research committee (Amano's institutional review board) and with the 1964 Helsinki declaration and its later amendments or comparable ethical standards.

Informed consent was obtained from all individual participants included in this study.

Open Access This article is distributed under the terms of the Creative Commons Attribution 4.0 International License (http:// creativecommons.org/licenses/by/4.0/), which permits unrestricted use, distribution, and reproduction in any medium, provided you give appropriate credit to the original author(s) and the source, provide a link to the Creative Commons license, and indicate if changes were made.

\section{References}

1. Flammer J, Mozaffarieh M (2007) What is the present pathogenetic concept of glaucomatous optic neuropathy? Surv Ophthalmol 52(suppl 2):S162-S173

2. Yarmohammadi A, Zangwill LM, Dinizz-Filho A et al (2017) Peripapillary and macular vessel density in patients with glaucoma and single-hemifield visual field defect. Ophthalmology 124:709-719

3. Lee EJ, Lee KM, Lee SM, Kim TW (2016) OCT angiography of the peripapillary retina in primary open angle glaucoma. Invest Ophthalmol Vis Sci 57:6265-6270

4. Bojikian K-D, Chen C-L, Wen JC et al (2016) Optic disc perfusion in primary open angle and normal tension glaucoma eyes using optical coherence tomography-based microangiography. PLoS One 11:e0154691. https://doi.org/10.1371/journal.pone.0154691 eCollection 2016

5. Wang L, Cull GA, Piper C, Burgoyne CF, Fortune B (2012) Anterior and posterior optic nerve head blood flow in nonhuman primate experimental glaucoma model measured by laser speckle imaging technique and microsphere method. Invest Ophthalmol Vis Sci 53:8303-8309

6. Liu L, Jia Y, Takasagawa HL et al (2015) Optical coherence tomography angiography of the peripapillary retina in glaucoma. JAMA Ophthalmol 133:1045-1052

7. Chihara E, Dimitrova G, Amano H, Chihara T (2017) Discriminatory power of superficial vessel density and prelaminar vascular flow index in eyes with glaucoma and ocular hypertension and normal eyes. Invest Ophthalmol Vis Sci 58:690-698

8. Tanihara H, Inoue T, Yamamoto T, Kuwayama Y, Abe H, Araie M (2013) Phase I clinical trials of a selective Rho-kinase inhibitor, K-115. JAMA Ophthalmol 131:1288-1295 
9. Tanihara H, Inoue T, Yamamoto T, Kuwayama Y, Abe H, Araie M (2013) Phase 2 randomized clinical study of a Rho-kinase inhibitor, $\mathrm{K}-115$, in primary open-angle glaucoma and ocular hypertension. Am J Ophthalmol 156:731-736

10. Shimokawa H, Takeshita A (2005) Rho-kinase is an important therapeutic target in cardiovascular medicine. Arterioscler Thromb Vasc Biol 25:1767-1777

11. Masumoto A, Mohri M, Shimokawa H, Urakami L, Usui M, Takeshita A (2002) Suppression of coronary artery spasm by the rho-kinase inhibitor fasudil in patients with vasospastic angina. Circulation 105:1545-1547

12. Sugiyama T, Shimizu E, Nakamura H et al (2016) Short term efficacy of ripasudil on intraocular pressure and optic nerve head blood flow in primary open angle glaucoma. Atarashii Ganka 33:11911195

13. Tokushige H, Waki M, Takayama Y, Tanihara H (2011) Effect of Y-39983, a selective rho-associated protein kinase inhibitor, on blood flow in optic nerve head in rabbits and axonal regeneration of retinal ganglion cells in rats. Curr Eye Res 36:964-970

14. Yamamoto K, Maruyama K, Himori N et al (2014) The novel Rho kinase (ROCK) inhibitor K-115: a new candidate drug for neuroprotective treatment in glaucoma. Invest Ophthalmol Vis Sci 55: 7126-7136

15. Wheeler L, Woldemussie E, Lai R (2003) Role of alpha-2 agonists in neuroprotection. Surv Ophthalmol 48(suppl1):S47-S51

16. Bhandari A, Cioffi GA, Van Buskirk EM, Orguel S, Wang L (1999) Effect of brimonidine on optic nerve blood flow in rabbits. Am J Ophthalmol 128:601-605

17. Jia Y, Wei E, Wang X et al (2014) Optical coherence tomography angiography of optic disc perfusion in glaucoma. Ophthalmology 121:1322-1332

18. Tan O, Chopra V, Lu AT et al (2009) Detection of macular ganglion cell loss in glaucoma by Fourier-domain optical coherence tomography. Ophthalmology 116:2305-2314

19. Zhang M, Hwang TS, Campbell JP et al (2016) Projection-resolved optical coherence tomographic angiography. Biomed Optics Express 7:816-828

20. Jia Y, Morrison JC, Tokayer J et al (2012) Quantitative OCT angiography of optic nerve head blood flow. Biomed Opt Express 3: 3127-3137

21. Yaoeda K, Shirakashi M, Fukushima A et al (2003) Relationship between optic nerve head microcirculation and visual field loss in glaucoma. Acta Ophthalmol Scand 81:253-259

22. Liu CJ-I, Ko Y-C, Cheng C-Y, Chou JC, Hsu W-M, Liu J-H (2002) Effect of latanoprost $0.005 \%$ and brimonidine tartrate $0.2 \%$ on pulsatile ocular blood flow in normal tension glaucoma. Br J Ophthalmol 86:1236-1239

23. Langham ME, To'mey K (1978) A clinical procedure for measuring the ocular pulse-pressure relationship and the ophthalmic arterial pressure. Exp Eye Res 27:17-25
24. Dimitrova G, Chihara E, Takahashi H, Amano H, Okazaki K (2017) Quantitative retinal optical coherence tomography angiography in patients with diabetes without diabetic retinopathy. Invest Ophthalmol Vis Sci 58:190-196

25. Liang Y, Downs JC, Fortune B, Cull G, Cioffi GA, Wang L (2009) Impact of systemic blood pressure on the relationship between intraocular pressure and blood flow in the optic nerve head of nonhuman primates. Invest Ophthalmol Vis Sci 50:2154-2160

26. Amano M, Fukata Y, Kabuki K (2000) Regulation and functions of Rho-associated kinase. Exp Cell Res 261:44-51

27. Honjo M, Tanihara H, Inatani M et al (2001) Effects of Rhoassociated protein kinase inhibitor Y-27632 on intraocular pressure and outflow facility. Invest Ophthalmol Vis Sci 42:137-144

28. Yamaguchi M, Nakao S, Arita R et al (2016) Vascular normalization by ROCK inhibitor: therapeutic potential of Ripasudil (K-115) eye drop in retinal angiogenesis and hypoxia. Invest Ophthalmol Vis Sci 57:2264-2276

29. Nakabayashi S, Kawai M, Yoshioka T et al (2015) Effect of intravitreal Rho kinase inhibitor ripasudil (K-115) on feline retinal microcirculation. Exp Eye Res 139(OCT):132-135

30. Isobe T, Kasai T, Kawai H (2016) Ocular penetration and pharmacokinetics of ripasudil following topical administration to rabbits. $\mathrm{J}$ Ocul Pharmacol Ther 32:405-412

31. Isobe T, Mizuno K, Kaneko Y, Ohta M, Koide T, Tanabe S (2014) Effects of K-115, a Rho-kinase inhibitor, on aqueous humor dynamics in rabbits. Curr Eye Res 39:813-822

32. Okinami S, Ohkuma M, Tsukahara I (1976) Kuhnt intermediary tissue as a barrier between the optic nerve and retina. Albrecht von Graefes Arch Clin Exp Ophthalmol 201:57-67

33. Schmidl D, Garhofer G, Schmetterer I (2011) The complex interaction between ocular perfusion pressure and ocular blood flowrelevance for glaucoma. Exp Eye Res 93:141-155

34. Wang L, Cull G, Burgoyne CF, Thompson S, Fortune B (2014) Longitudinal alterations in the dynamic autoregulation of optic nerve head blood flow revealed in experimental glaucoma. Invest Ophthalmol Vis Sci 55:3509-3516

35. Krupin T, Liebmann J, Greenfield DS, Ritch R, Gardiner S, LowPressure Glaucoma Study Group (2011) A randomized trial of brimonidine versus timolol in preserving visual function: results from the Low-Pressure Glaucoma Treatment Study. Am J Ophthalmol 151:671-681

36. Choi W, Moult EM, Waheed NK et al (2015) Ultrahigh-speed swept source optical coherence tomography angiography in nonexudative age-related macular degeneration with geographic atrophy. Ophthalmology 122:2532-2544

37. Geijer C, Bill A (1979) Effect of raised intraocular pressure on retinal prelaminar and retrolaminar optic nerve blood flow in monkey eyes. Invest Ophthalmol Vis Sci 18:1030-1042 